\begin{tabular}{|c|c|c|c|}
\hline$D E$ & \multirow{3}{*}{$\begin{array}{l}\text { DE GRUYTER } \\
\text { OPEN }\end{array}$} & $\begin{array}{l}\text { HUNGARIAN JOURNAL OF } \\
\text { INDUSTRY AND CHEMISTRY }\end{array}$ & \multirow[t]{3}{*}{ 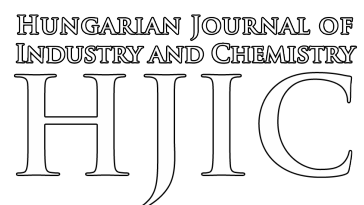 } \\
\hline & & Vol. 44(2) pp. 105-111 (2016) & \\
\hline & & $\begin{array}{l}\text { hjic.mk.uni-pannon.hu } \\
\text { DOI: } 10.1515 / \text { hjic-2016-0013 }\end{array}$ & \\
\hline
\end{tabular}

\title{
MODELLING A THREE-PHASE CURRENT SOURCE INVERTER
}

\author{
LÁsZló NEUKIRCHNER* AND ATTILA MAGYAR \\ Department of Information Technology, University of Pannonia, Egyetem u. 10, Veszprém, 8200, \\ HUNGARY
}

\begin{abstract}
A current source inverter model has been developed in the given paper that is constructed from six LTI models for the different switching modes. The overall model is in a piecewise affine form that supports the use of model predictive control. The model has been verified against engineering expectations and its open-loop performance shows that it is a promising basis of model predictive control structures.
\end{abstract}

Keywords: model predictive control, current source inverter, space vector modulation, modelling, simulation

\section{Introduction}

In industrial applications power electronic converters are divided into two major classes, namely voltage source (VSI) and current source inverters (CSI). As a VSI employs modulating the voltage wave through the inverter's legs, its counterpart relies on modulated current waveforms. To operate a VSI the inverter's switching devices should operate in a specified alternating modulation to avoid shorting the inverter's legs, while its current source counterpart could embed this operation into the modulation. Thyristor rectifiers suffer from several drawbacks, e.g. high-input current harmonic distortion or a low power factor. Pulse width modulated current-source inverters, on the other hand, are free from the above-mentioned drawbacks. In such cases, a smoothing LC filter is placed on the AC side and a choke inductor DC side to reduce the harmonic injection resulting from the current modulating PWM operation. In the case of sinusoidal supply voltages, an input power factor with unit value can be achieved if both requirements are met. In conventional control structures, PWM-CSR is usually operated by off-line patterns and look-up tables like in trapezoidal modulation and selective harmonic elimination. The use of these techniques simplifies the calculation of the gate control signals with the special requirements of applied switches like IGBTs. Input filtering, on the other hand, may result in oscillatory transient behaviour. This can be attenuated by adding damping resistive elements, but using them also reduces the overall efficiency. Space vector modulation (SVM) has proven to result in a high voltage gain, reduced switching frequency and low

*Correspondence: neukirchner.laszlo@uni-pannon.hu current harmonic distortion, and could be straightforwardly implemented in digital systems [1]. In the second section, the structure of the investigated inverter is presented. In the next section, a detailed open-loop model is presented, and in the fourth section a predictive strategy is chosen for controlling SVM.

\section{Inverter Structure}

The current source inverter normally requires threephase inductive and capacitive filtering $\left(L_{\mathrm{f}}\right.$ and $C_{\mathrm{f}}$, respectively), improving the load current and voltage waveform's harmonic distortion, and in thermistor appliances assisting the commutation of the switching devices. The legs of the device should include protective diodes, otherwise the absence of a current path introduces a high-voltage spike, causing damage to the switching devices [2]:

- The switching devices used are symmetrical, they do not require freewheeling diodes.

- The current source inverter produces a threephase pulse-width modulated current instead of a pulse-width modulated voltage. As such, a capacitor needs to be installed on the output for filtering purposes. The high voltage derivative is associated with the VSI.

- The rate at which the DC current rises is limited by the DC choke, allowing sufficient time for the protection circuit to function in the case of a short circuit at the inverter output terminals.

- As a restraint, the DC link current cannot be instantaneously changed, which resulted in a reduction in dynamic performance. 


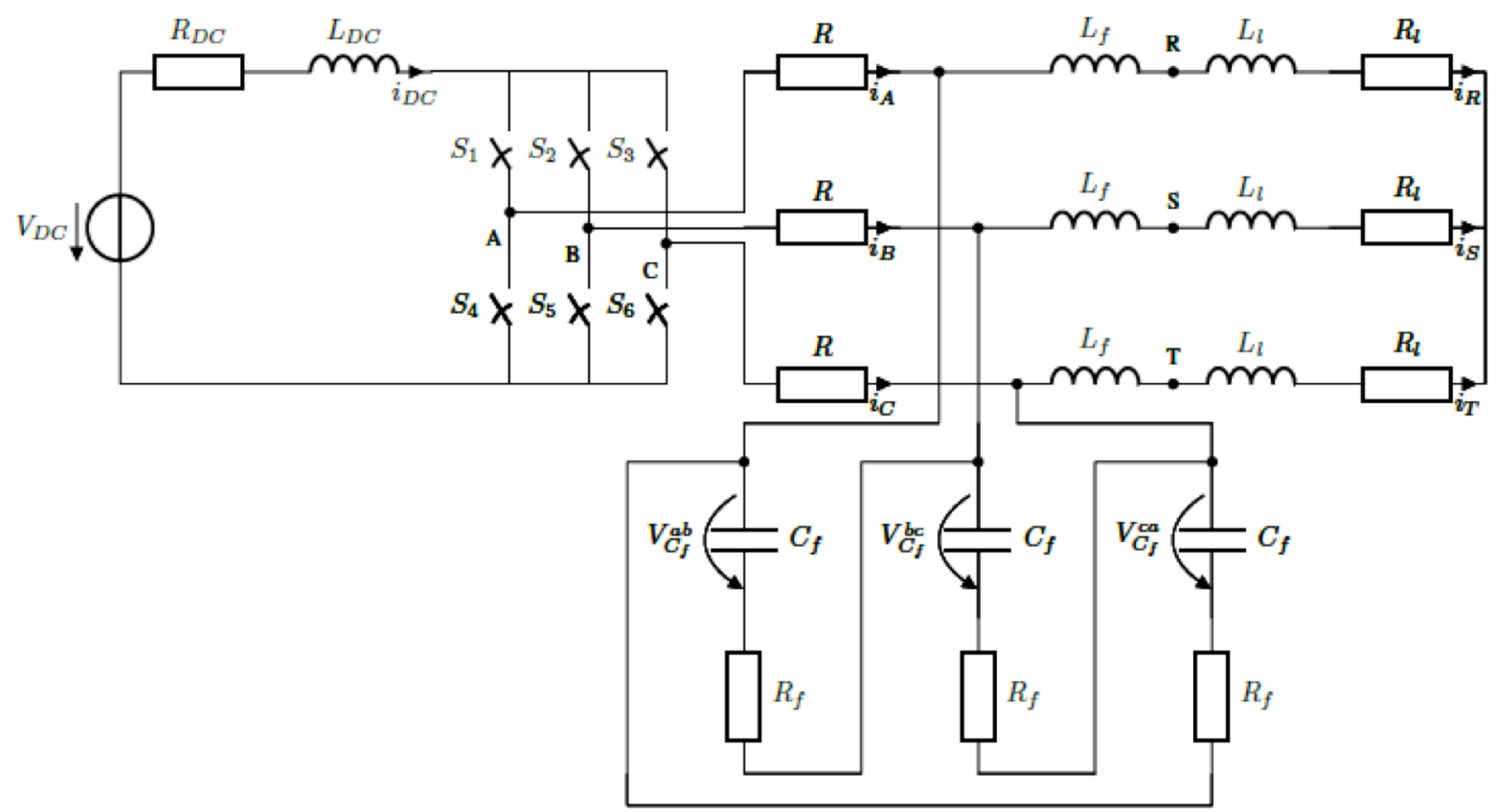

Figure 1. Current source inverter with an LC filter and RL load.

\section{Open-Loop Switched Model with Space Vector Modulation}

\subsection{Space Vector Formulation}

The switched model of the three-phase CSI supplying a three-phase load through an LC filter and RL load is shown in Fig.1. The LC filter is a low-pass filter included with the three-phase CSI to decrease the THD by eliminating higher order harmonics. The implemented LC filter includes a resistor $R_{\mathrm{f}}$ in series with a capacitor $C_{\mathrm{f}}$. This resistor provides a passive damping solution and it was recommended to be within the range of 0.5 to $1 \Omega$. Moreover, the resistor RDC represents any start-up pre-charging resistance or any resistance in series with the DC voltage source VDC. For constructing the model the following assumptions are made:

- The LC filter and the RL load's circuit components are symmetric.

- Only a continuous conduction mode is assumed.

- Each time, the switch state index s is known.

- The switching devices used for modulation are assumed to be ideal, with zero losses and switching is considered to be instant.

- No external disturbance is assumed.

- No magnetic coupling between the inductances is assumed.

With the above assumptions, the equation system of the inverter, filter and load for all the switching states can be created, as different state-space models [3, 4]. Under the above-mentioned constraints, the three-phase current source inverter has nine switching states as can be seen in Table 1.
Table 1. Switching states of SVPWM modulated CSI.

\begin{tabular}{cccccc}
\hline & $\begin{array}{c}\text { active } \\
\text { switches }\end{array}$ & $i_{\mathrm{A}}$ & $i_{\mathrm{B}}$ & $i_{\mathrm{C}}$ & $\begin{array}{c}\text { Space } \\
\text { vector }\end{array}$ \\
\hline Zero & $S_{1}, S_{4}$ & & & & \\
States & $S_{2}, S_{5}$ & 0 & 0 & 0 & $\boldsymbol{I}_{0}$ \\
& $S_{3}, S_{6}$ & & & & \\
\hline \multirow{5}{*}{ Active } & $S_{6}, S_{1}$ & $i_{\mathrm{DC}}$ & $-i_{\mathrm{DC}}$ & 0 & $\boldsymbol{I}_{1}$ \\
& $S_{1}, S_{2}$ & $i_{\mathrm{DC}}$ & 0 & $-i_{\mathrm{DC}}$ & $\boldsymbol{I}_{2}$ \\
States & $S_{2}, S_{3}$ & 0 & $i_{\mathrm{DC}}$ & $-i_{\mathrm{DC}}$ & $\boldsymbol{I}_{3}$ \\
& $S_{3}, S_{4}$ & $-i_{\mathrm{DC}}$ & $i_{\mathrm{DC}}$ & 0 & $\boldsymbol{I}_{4}$ \\
& $S_{4}, S_{5}$ & $-i_{\mathrm{DC}}$ & 0 & $i_{\mathrm{DC}}$ & $\boldsymbol{I}_{5}$ \\
& $S_{5}, S_{6}$ & 0 & $-i_{\mathrm{DC}}$ & $i_{\mathrm{DC}}$ & $\boldsymbol{I}_{6}$ \\
\hline
\end{tabular}

These switching states can be classified as zero switching states $\left(\mathrm{I}_{0}\right)$ and active switching states $\left(\boldsymbol{I}_{1}, \ldots\right.$, $\left.\boldsymbol{I}_{6}\right)$. In addition, there are three zero states which implies vertical conduction of the inverter leg. This implies that the source is shorted, leading to $i_{\mathrm{A}}=i_{\mathrm{B}}=i_{\mathrm{C}}=0$. This operating mode is often referred to as the bypass operation. Assuming that the operation of the inverter is three-phase balanced then Eq.(1) applies

$$
i_{\mathrm{A}}+i_{\mathrm{B}}+i_{\mathrm{C}}=0
$$

According to Fig.2, the five active vectors can be expressed as:

$$
\boldsymbol{I}_{\mathrm{k}}=\frac{2}{\sqrt{3}} I_{\mathrm{DC}} \mathrm{e}^{\mathrm{j}\left((\mathrm{k}-1) \frac{\pi}{3}-\frac{\pi}{6}\right)}, k=1,2, \ldots, 6
$$

The active and zero vectors are assumed to be stationary, on the contrary, the current reference vector $\boldsymbol{I}_{\text {ref }}$ in Fig. 2 rotates with an angular velocity $\omega=2 \pi f$, and $\theta(t)=\int_{0}^{t} \omega(t) d t+\theta(0)$, where $f$ is the fundamental frequency of the inverter output current and $\theta$ is the angular displacement. A desired vectorial length and angle, $\boldsymbol{I}_{\text {ref }}$ can be calculated from the neighbouring space 


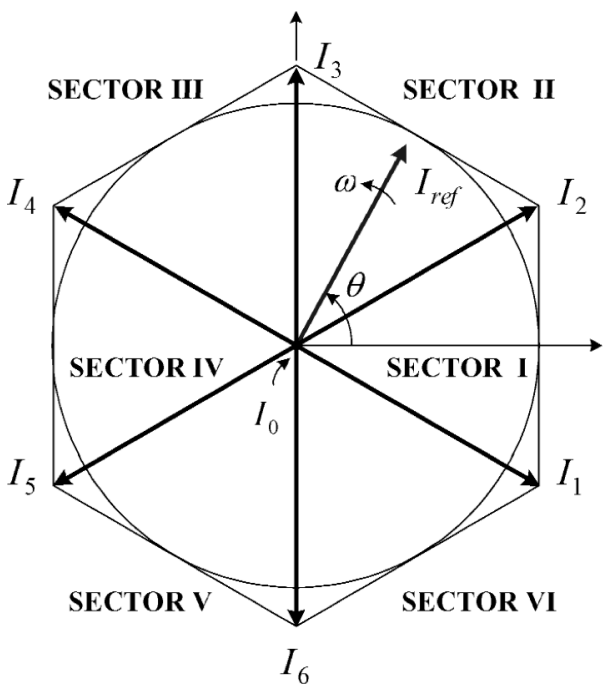

Figure 2. A typical space vector diagram for the CSI. $\boldsymbol{I}_{1}, \ldots, \boldsymbol{I}_{6}$ are the active vectors, $\boldsymbol{I}_{0}$ is the zero vector, $\omega$ is the angular velocity, $\theta$ is the angular displacement and the Cartesian coordinate system represents the imaginary and real axes. The active vectors form a symmetric hexagon with six identical sectors, while the zero vector $\boldsymbol{I}_{0}$ lies at the center of the hexagon.

vectors (Fig.3), based on the switching pattern and frequency of the inverter. As $\boldsymbol{I}_{\text {ref }}$ passes through the vectorial spaces, different switch sets are applied to formulate the desired output. As a result, when $\boldsymbol{I}_{\text {ref }}$ rotates one revolution, the output continues for one cycle based on the fundamental frequency.

\subsection{Calculating Dwell-Time}

The dwell time for stationary vectors represents the time that space vectors spend in a sector during $T_{\mathrm{S}}$. The basic method used for dwell-time calculations is the so-called ampere-second balancing principle. The main idea behind it is that the product of the sampling period $T_{\mathrm{S}}$ and the reference vector $\boldsymbol{I}_{\text {ref }}$ is equal to the sum of the current vectors multiplied by the time interval of chosen space vectors. With small enough $T_{\mathrm{S}}, \boldsymbol{I}_{\text {ref }}$ can be assumed as constant over a period. With this notion, the reference current is approximated from the neighbouring vectors corresponding to the actual sector and from the zero vector for adjusting the length.

The ampere-second balancing principle is given by

$$
\begin{array}{ccc}
\boldsymbol{I}_{\mathrm{ref}} T_{\mathrm{S}} & = & \boldsymbol{I}_{1} T_{1}+\boldsymbol{I}_{2} T_{2}+\boldsymbol{I}_{0} T_{0} \\
T_{\mathrm{S}} & = & T_{1}+T_{2}+T_{0}
\end{array}
$$

where $T_{0}, T_{1}$, and $T_{2}$ denote the dwell times of the vectors $\boldsymbol{I}_{0}, \boldsymbol{I}_{1}$, and $\mathbf{I}_{2}$. From Eq.(2), the individual values of space vectors in SECTOR I are defined in Eq.(4):

$$
\begin{aligned}
& \boldsymbol{I}_{1} T_{\mathrm{S}}=\frac{2}{\sqrt{3}} I_{\mathrm{DC}} \mathrm{e}^{-\mathrm{j} \frac{\pi}{6}} \\
& \boldsymbol{I}_{2} T_{\mathrm{S}}=\frac{2}{\sqrt{3}} I_{\mathrm{DC}} \mathrm{e}^{\mathrm{j} \frac{\pi}{6}} \\
& \boldsymbol{I}_{0} T_{\mathrm{S}}=c
\end{aligned}
$$

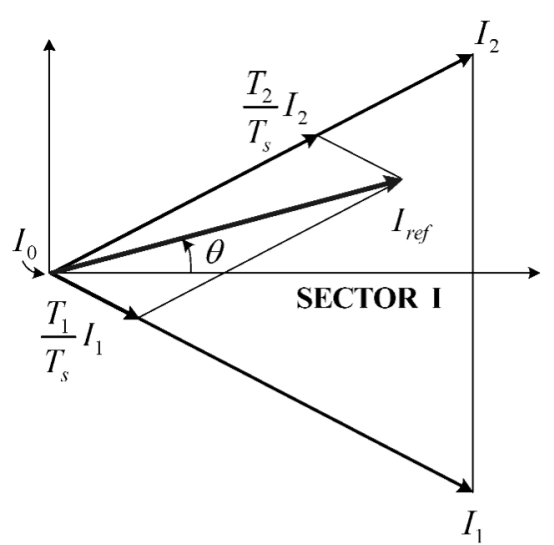

Figure 3. Calculation of $\boldsymbol{I}_{\text {ref }}$ in Sector I, where the Cartesian coordinate system represents the imaginary and real axes.

Projecting the resulting complex vector onto the real and imaginary axes leads to:

$$
\begin{array}{lll}
\boldsymbol{I}_{\text {ref }} \cos (\theta) T_{\mathrm{S}} & = & I_{\mathrm{DC}}\left(T_{1}+T_{2}\right) \\
\boldsymbol{I}_{\text {ref }} \sin (\theta) T_{\mathrm{S}} & = & \frac{1}{\sqrt{3}} I_{\mathrm{DC}}\left(-T_{1}+T_{2}\right) .
\end{array}
$$

Solving Eqs.(3) and (5) yields the dwell times $T_{0}$, $T_{1}$, and $T_{2}$ at any given $\theta$ angle as shown in Eq.(6):

$$
\begin{gathered}
T_{1}=m_{\mathrm{a}} \sin \left(\frac{\pi}{6}-\theta\right) T_{\mathrm{S}} \\
T_{2}=m_{\mathrm{a}} \sin \left(\frac{\pi}{6}+\theta\right) T_{\mathrm{S}} \\
T_{0}=\quad T_{\mathrm{S}}-T_{1}-T_{2} \\
\text { for } \quad-\frac{\pi}{6} \leq \theta \leq \frac{\pi}{6},
\end{gathered}
$$

where $m_{\mathrm{a}}=\left|\boldsymbol{I}_{\text {ref }}\right| / I_{\mathrm{DC}}$ is the modulation index in the domain: $m_{\mathrm{a}} \in[0,1]$.

When $\boldsymbol{I}_{\text {ref }}$ is inside of other sectors a multiple of $\pi / 3$ is subtracted from $\theta$ so that the modified angle $\sigma$ happens to fall within the range of $-\frac{\pi}{6} \leq \theta \leq \frac{\pi}{6}$ for use in Eq.(7):

$$
\begin{array}{ccc}
\sigma & =\theta-(k-1) \frac{\pi}{3} \\
s & =1,2, \ldots, 6 \\
\text { for } & -\frac{\pi}{6} \leq \theta \leq \frac{\pi}{6},
\end{array}
$$

where $s$ is the sector index. This method save sthe need for implementing unnecessary calculations and code capacities.

\subsection{Switching Sequence}

As far as designing the switching pattern of the SVM for the CSI a few notions should be specified for switching loss reduction:

- The state transition should involve the least amount of switches (one switch is desired).

- Minimum amount of switches should be involved as $\boldsymbol{I}_{\text {ref }}$ transits from the current to the next state. 
Table 2. Typical switching sequence for Sector I. The sequence was repeated twice to achieve a smoother output signal. In this case, the sampling period $T_{\mathrm{S}}$ is one $12^{\text {th }}$ of the fundamental time period. The active switch pairs are $S_{6}, S_{1}$ and $S_{1}, S_{2}$ for space vectors $\boldsymbol{I}_{1}$ and $\boldsymbol{I}_{2}$. The chosen zero state (with switches $S_{1}, S_{4}$ ) is chosen for $\boldsymbol{I}_{0}$ such that the minimum switching loss requirement is met.

\begin{tabular}{ccccccc}
\hline Vectors & $\boldsymbol{I}_{1}$ & $\boldsymbol{I}_{2}$ & $\boldsymbol{I}_{0}$ & $\boldsymbol{I}_{1}$ & $\boldsymbol{I}_{2}$ & $\boldsymbol{I}_{0}$ \\
\hline$S_{1}$ & $\mathrm{X}$ & $\mathrm{X}$ & $\mathrm{X}$ & $\mathrm{X}$ & $\mathrm{X}$ & $\mathrm{X}$ \\
$S_{2}$ & & $\mathrm{X}$ & & & $\mathrm{X}$ & \\
$S_{3}$ & & & & & & \\
$S_{4}$ & & & $\mathrm{X}$ & & & \\
$S_{5}$ & & & & & & \\
$S_{6}$ & $\mathrm{X}$ & & & $\mathrm{X}$ & & $\mathrm{X}$ \\
\hline dwell & $T_{1}$ & $T_{2}$ & $T_{0}$ & $T_{1}$ & $T_{2}$ & $T_{0}$ \\
time & & $T_{\mathrm{S}}$ & & & $T_{\mathrm{S}}$ & \\
\hline
\end{tabular}

The typical sequence can be observed in Table 2 . $\boldsymbol{I}_{\text {ref }}$ is created by $\boldsymbol{I}_{1}, \boldsymbol{I}_{2}$, and $\boldsymbol{I}_{0}$ with the mentioned dwell time rule $6 . T_{\mathrm{s}}$ can be divided into three or more segments $\left(T_{0}, T_{1}\right.$, and $\left.T_{2}\right)$ according to the desired THD value of the output current.

From the above, a continuous switching sequence for the SVPWM modulation can be obtained by Eq.(8):

$$
\begin{array}{ll}
\boldsymbol{I}_{\mathrm{k}}, \boldsymbol{I}_{\mathrm{k}+1}, \boldsymbol{I}_{0} & k=1,2, \ldots, 5 \\
\boldsymbol{I}_{\mathrm{k}}, \boldsymbol{I}_{1}, \boldsymbol{I}_{0} & k=6,
\end{array}
$$

where $k$ is the sector index.

\subsection{Obtaining Open-Loop 'Piecewise Affine' Model}

With every state vector $\boldsymbol{I}_{0}, \boldsymbol{I}_{1}, \ldots, \mathbf{I}_{6}$ a different switch configuration and a different circuit configuration is present at a given time. For every case the circuit's state equations can be written in continuous state-space form:

$$
\begin{aligned}
\dot{\boldsymbol{x}}(t) & =\mathbf{A}_{\mathrm{S}} \boldsymbol{x}(t)+\mathbf{B}_{\mathrm{S}} \boldsymbol{u}(t) \\
\dot{\boldsymbol{y}}(t) & =\mathbf{C}_{\mathrm{S}} \boldsymbol{x}(t) \\
\mathrm{S} & =0,1, \ldots, 6
\end{aligned}
$$

where $\mathbf{A}_{\mathrm{S}}$ is the $s^{\text {th }}$ state matrix, $\mathbf{B}_{\mathrm{S}}$ is the $s^{\text {th }}$ input matrix, $\mathbf{C}_{\mathrm{S}}$ is the $s^{\text {th }}$ output matrix, $\boldsymbol{x}(t)$ and $\boldsymbol{u}(t)$ are space vectors, and $s$ represents the space vector index with $s=$ 0 as the zero state and $s=1,2, \ldots, 6$ as the active states. Since all the equation systems have the same input $\left(\mathbf{B}_{\mathrm{S}}\right)$ and output $\left(\mathbf{C}_{\mathrm{S}}\right)$ matrices they will be referred to as $\mathbf{B}$ and $\mathbf{C}$ matrices.

Any linear circuit can be represented by a number of differential equations equal to the number of energy storage units in the circuit. However, in three-phase balanced circuits, the number of equations can be reduced by a third factor because any three-phase quantity can be expressed in terms of the other two phase quantities. Therefore, since currents flowing through $L_{\mathrm{f}}$ and $L_{\mathrm{l}}$ and voltages across $C_{\mathrm{f}}$ are three-phase balanced quantities only four linear differential equations representing the $\mathrm{AC}$ side and one linear differential equation representing DC current across $L_{\mathrm{DC}}$ are needed. Hence, linear differential equations for the state variable $\boldsymbol{x}=\left[i_{\mathrm{DC}} V_{\mathrm{C}_{\mathrm{f}}}^{\mathrm{ab}} V_{\mathrm{C}_{\mathrm{f}}}^{\mathrm{bc}} i_{\mathrm{R}} i_{\mathrm{S}}\right]^{\mathrm{T}}$, input variable $\boldsymbol{u}=$ $V_{\mathrm{DC}}$ and output variable $\boldsymbol{y}=\left[i_{\mathrm{R}} i_{\mathrm{S}}\right]^{\mathrm{T}}$ can be derived using KVL and KCL. Arranging the expressions for the aforementioned state variables and recognizing $V_{\mathrm{DC}}$ as the input of the system, yields the following state-space matrices for the zero switching state as shown in Eqs.(10)-(12):

$$
\begin{gathered}
\mathbf{A}_{\mathbf{0}}=\left[\begin{array}{ccccc}
-\frac{R_{\mathrm{DC}}}{L_{\mathrm{DC}}} & 0 & 0 & 0 & 0 \\
0 & -\frac{1}{Q_{\mathrm{a}}} & 0 & -\frac{R}{Q_{\mathrm{a}}} & \frac{R}{Q_{\mathrm{a}}} \\
0 & 0 & -\frac{1}{Q_{\mathrm{a}}} & -\frac{R}{Q_{\mathrm{a}}} & -\frac{2 R}{Q_{\mathrm{a}}} \\
0 & \frac{2 R}{Q_{\mathrm{c}}} & \frac{R}{Q_{\mathrm{c}}} & \frac{Q_{\mathrm{b}}}{Q_{\mathrm{a}}} & 0 \\
0 & \frac{R}{Q_{\mathrm{c}}} & \frac{R}{Q_{\mathrm{c}}} & 0 & \frac{Q_{\mathrm{b}}}{Q_{\mathrm{a}}}
\end{array}\right] \\
\mathbf{B}=\left[\begin{array}{ccccc}
-\frac{1}{L_{\mathrm{DC}}} & 0 & 0 & 0 & 0
\end{array}\right]^{\mathrm{T}} \\
\mathbf{C}=\left[\begin{array}{lllll}
0 & 0 & 0 & 1 & 0 \\
0 & 0 & 0 & 0 & 1
\end{array}\right],
\end{gathered}
$$

where $Q_{\mathrm{a}}=C\left(3 R+R_{\mathrm{f}}\right), Q_{\mathrm{b}}=R R_{\mathrm{f}}+3 R R_{1}+R_{\mathrm{f}} R_{\mathrm{l}}$ and $Q_{\mathrm{c}}$ $=\left(3 R+R_{\mathrm{f}}\right)\left(L_{\mathrm{f}}+L_{\mathrm{l}}\right)$.

\subsection{Open-Loop Performance}

As CSI exhibits different LTI regions for different state matrices $\boldsymbol{A}_{0}, \boldsymbol{A}_{1}, \ldots, \boldsymbol{A}_{6}$ according to the used space vector $\boldsymbol{I}_{0}, \boldsymbol{I}_{1}, \ldots, \boldsymbol{I}_{6}$ in the corresponding dwell time, the piecewise affine approach (PWA) can be used to describe the overall dynamics. The discrete time piecewise affine model can be obtained by discrediting the set of continuous affine models in the form of Eq.(13)

$$
\boldsymbol{x}_{\mathrm{p}+1}=\left\{\begin{array}{ccc}
\Phi_{1} \boldsymbol{x}_{\mathrm{p}}+\boldsymbol{\Gamma} \boldsymbol{u}_{\mathrm{p}}, & \text { if } & {\left[\begin{array}{l}
\boldsymbol{u}_{1} \\
\boldsymbol{x}_{1}
\end{array}\right] \in P_{1}} \\
& \vdots & \\
\Phi_{S} \boldsymbol{x}_{\mathrm{p}}+\Gamma \boldsymbol{u}_{\mathrm{p}} & \text { if } & {\left[\begin{array}{l}
\boldsymbol{u}_{\mathrm{s}} \\
\boldsymbol{x}_{\mathrm{s}}
\end{array}\right] \in P_{S}}
\end{array}\right.
$$

Such systems are composed of $s$ local affine models whose parameters in $\left(\boldsymbol{\Phi}_{\mathrm{s}}\right)$ change according to which polyhedron $P_{\mathrm{k}}$ contains the state vector.

The simulated waveforms for $\mathrm{VDC}=400 \mathrm{~V} \mathrm{DC}$ link with pre-charge resistance $R_{\mathrm{DC}}=1 \Omega$ and choke inductance $L_{\mathrm{DC}}=2 \mathrm{e}^{-1} H$ using the space vector modulation as shown in Fig.5, where $i_{\mathrm{A}}$ denotes the PWM current of the inverter and $i_{\mathrm{R}}$ stands for the phase current through the load. The inverter operates at a fundamental frequency of $f=50 \mathrm{~Hz}$. The filter capacitor $C f=20 \mathrm{e}^{-6} F$, the filter inductance $L_{\mathrm{f}}=10 \mathrm{e}^{-3} H$ with filter resistance $R_{\mathrm{f}}=1 \Omega$ and $R=1 \Omega$ representing losses. The inverter consists of three-phase balanced inductive load $L_{1}=2 \mathrm{e}^{-3}$ and resistive load $R_{1}=5 \Omega$. The open-loop performance can be observed in Fig.4 and the modulation and PWM formulation in Fig.5. 


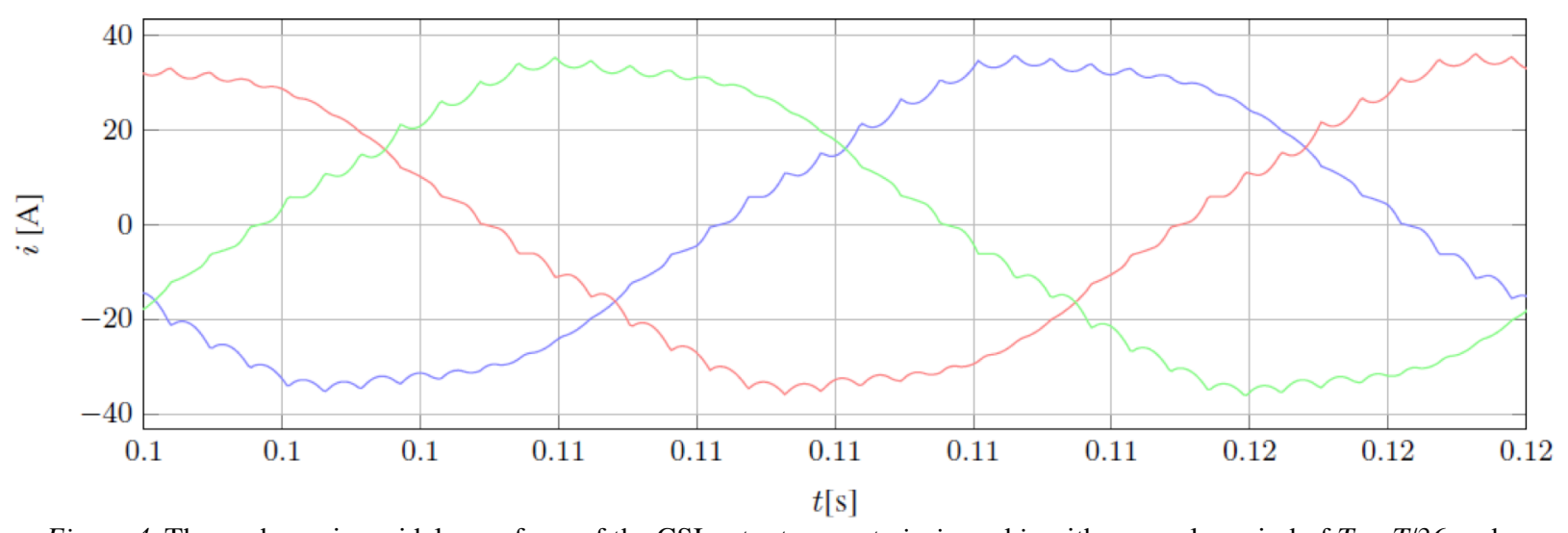

Figure 4. Three-phase sinusoidal waveform of the CSI output currents $i_{\mathrm{A}}, i_{\mathrm{B}}$, and $i_{\mathrm{C}}$ with a sample period of $T_{\mathrm{s}}=T / 36$ and a modulation index of $m_{\mathrm{a}}=0.9$. The output wave can be smoothened by adding higher filter parameters or further reducing the sampling period.

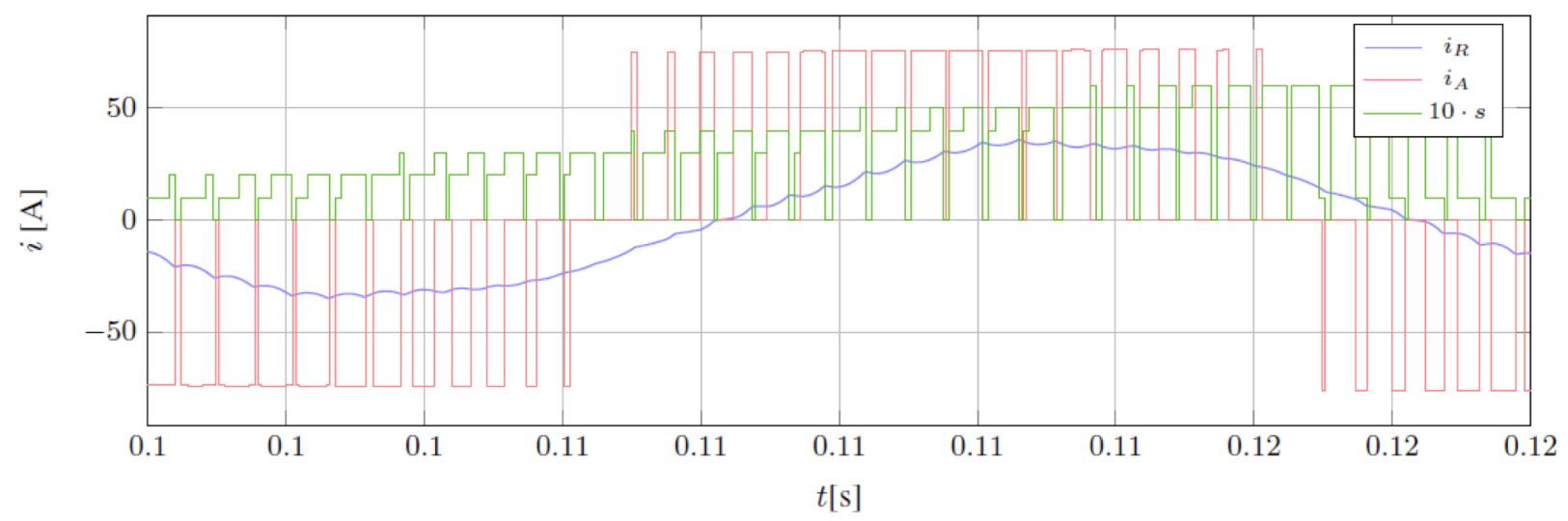

Figure 5. Comparison of the inverter output current $i_{\mathrm{A}}$ and the filtered load current $i_{\mathrm{R}}$ with $T_{\mathrm{s}}=T / 36$ and $m_{\mathrm{a}}=0.9$. The background $s$ 's value indicates the corresponding affine state of the model at a given time.

\section{MPC assisted SVPWM}

The aim of the previous sections was to develop a model for the inverter that describes the switching behaviour of the dynamics. The obtained piecewise affine model gives a good basis for the model predictive control technique that is advantageous from two points of view: the possibility of introducing constraints to the state and input variables and the fact that optimal control problems can be formulated within it.

The aim is to find the optimal space vectors in such a way that the error between the predicted states of the inverter and the chosen reference values are minimized with respect to constraints. Previously, the method of model predictive control was reserved only for applications with relatively slow time scales, like plants and chemical processes. The reason for this is that MPC requires an optimization problem to be solved at each sampling instant in order to obtain the control input. Over the past decade, more and more applications use fast MPC applications, thanks to the advances in computer technology. The optimization problem is often NP hard, and finding a solution is taxing in terms of the calculation time of the control device, thus limiting the rate at which the control could intervene with the application. In particular, model predictive control has been applied in the field of power electronics, where the control frequency ranges from a few hundred $\mathrm{Hz}$ up to a few $\mathrm{MHz}[5,6]$. The main challenge of implementing MPC consists of solving the associated optimization problem in a bounded time.

The predicted values are assumed to be constant after time $k$. Then, the control problem can be formulated as follows:

$\min J(\boldsymbol{x}[k])$

s.t. $\boldsymbol{x}[k]=\mathbf{x}[k \mid k]$

$J(\boldsymbol{x}[k])=\sum_{\mathrm{j}=1}^{\mathrm{N}}\left\|Q\left(\boldsymbol{x}[k+j \mid k]-\boldsymbol{x}_{\mathrm{ref}}([k])\right)\right\|^{2}$

where $\boldsymbol{x}[k+j \mid k]$ denotes the prediction of the state at time $k+\mathrm{j}$ based on the information available at time $k$, $N$ is the prediction horizon, $Q$ is the constant weighting. The optimal state-finding algorithm follows:

OptimalError $=$ inf

for $s=1: 7$ do

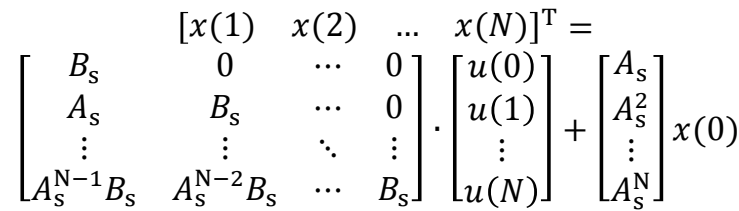




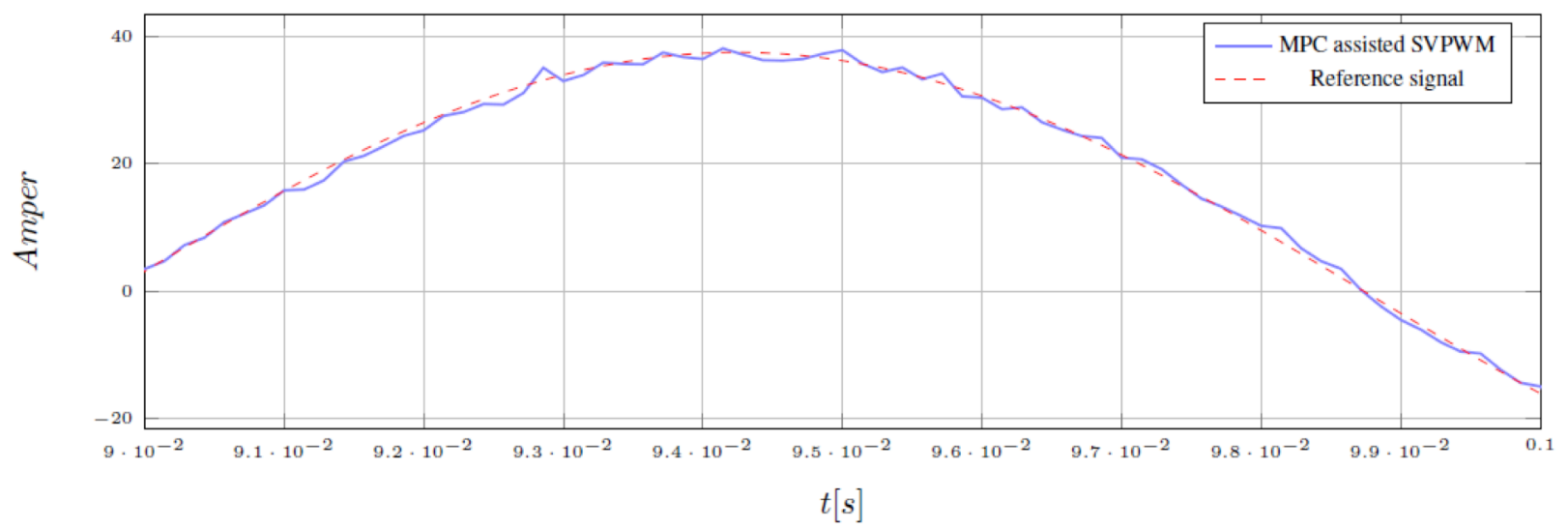

Figure 6. MPC reference tracking with $f=50 \mathrm{~Hz}, \hat{I}=37.5 \mathrm{~A}$, and $N=2$. In order to create the simplest implicit MPC for a finite state PWA environment, every affine state was tested with the according horizon in every iteration.

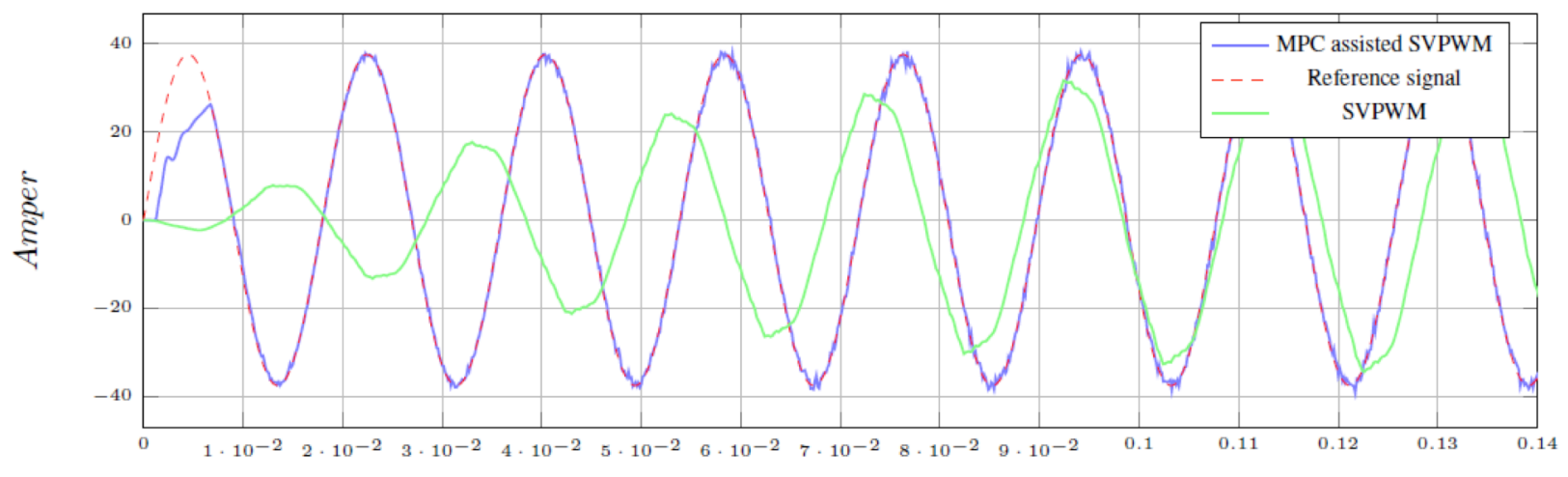

$t[s]$

Figure 7. Comparison of classic SVPWM and MPC on the same system. The SVPWM is applied with a modulation index of 0.9 , and the MPC reference tracking with $f=50 \mathrm{~Hz}, \hat{I}=37.5 \mathrm{~A}$, and $N=2$.

$$
\begin{aligned}
& \text { Error }_{\mathrm{s}}=J_{\mathrm{s}}(x) \\
& \text { if Error } \\
& \text { OptimalError }_{\mathrm{s}}=\text { Error }_{\mathrm{s}} \\
& \text { Optimal_s }=s
\end{aligned}
$$

where OptimalError represents the minimal error value, Error_s is the actual error value in each iteration, and Optimal_s is the optimal state. The algorithm uses a parallel implicit MPC scheme for every state. Since there are only seven states, with a low horizon the algorithm can be feasibly implemented. When $f=50 \mathrm{~Hz}$, $\hat{I}=37.5 \mathrm{~A}$, and $N=2$, deviations from the optimal trajectory are present during optimization as shown in Fig.6, but they are still acceptable in terms of harmonic distortion. The performance observed in Fig.7 can be compared to the open-loop solution. It can be stated that due to the rigid structure of the open-loop modulation's rigid structure the dynamical properties of the MPCassisted system exhibit better performance in terms of time response and signal shape.

\section{Conclusion}

A current source inverter model has been developed in this paper that is constructed from six LTI models for the different switching modes using SVPWM modulation. The obtained overall model is in a piecewise affine form that supports the use of model predictive control in the future. The model has been verified against engineering expectations and its openloop performance shows that it is a promising basis of further work in terms of identification and control. As the next step, a model predictive control synthesis was performed based on the technology-driven optimality criteria to assist the open-loop SVPWM modulated system. Due to the implicit MPC-assisted modulation the optimal space vector could be found and utilized in each state over an acceptable timescale due to the low horizon. As such a significantly lower time response and better signal shape was achieved.

\section{SYMBOLS}

$\begin{array}{ll}\text { A } & \text { state matrix } \\ \mathbf{B} & \text { input matrix } \\ \mathbf{C} & \text { output matrix } \\ C_{\mathrm{f}} & \text { filter capacitance } \\ f & \text { fundamental frequency } \\ \boldsymbol{\Phi} & \text { discrete state matrix } \\ \boldsymbol{\Gamma} & \text { discrete input matrix } \\ \boldsymbol{I}_{0} \boldsymbol{I}_{1}, \ldots, \boldsymbol{I}_{6} & \text { space vector }\end{array}$

$i_{\mathrm{A}}, i_{\mathrm{B}}, i_{\mathrm{C}}$ inverter output currents per phase 


$\begin{array}{ll}i_{\mathrm{DC}} & \text { DC link current } \\ i_{\mathrm{R}}, i_{\mathrm{S}}, i_{\mathrm{T}} & \text { load currents per phase } \\ \boldsymbol{I}_{\text {ref }} & \text { reference current value } \\ k & \text { sector index } \\ L_{\mathrm{f}} & \text { filter inductance } \\ L_{\mathrm{l}} & \text { load inductance } \\ \mathrm{LTI} & \text { Linear Time Invariant } \\ m_{\mathrm{a}} & \text { modulation index } \\ \mathrm{MPC} & \text { Model Predictive Control } \\ v_{\mathrm{cr}} & \text { triangular carrier wave for TPWM } \\ v_{\mathrm{m}} & \text { modular wave for TPWM } \\ p & \text { discrete time index } \\ P & \text { space vector containing polyhedron } \\ \mathrm{PWA} & \text { 'Piecewise Affine' Current Source Inverter } \\ & \text { (CSI) } \\ \mathrm{PWM} & \text { Pulse Width Modulation } \\ R & \text { loss resistance } \\ R_{\mathrm{DC}} & \text { DC link start-up pre-charging resistance } \\ R_{\mathrm{f}} & \text { filter resistance } \\ R_{\mathrm{l}} & \text { load resistance } \\ S & \text { space vector index } \\ S_{1}, S_{2}, \ldots, & S_{6} \text { Switching devices } \\ \sigma & \text { modified angle } \\ \mathrm{SHE} & \text { Selective Harmonic Elimination } \\ \mathrm{SVPWM} & \text { Space Vector Pulse Width Modulation } \\ T & \text { fundamental cycle time } \\ T_{\mathrm{k}} & \text { dwell time of corresponding space vectors } \\ T_{\mathrm{s}} & \text { sampling period } \\ \mathrm{TPWM} & \text { Trapezoidal Pulse Width Modulation } \\ \theta & \text { angular displacement } \\ \boldsymbol{u} & \text { input vector } \\ V_{\mathrm{DC}} & \text { DC link voltage source value } \\ V_{\mathrm{Cb}}^{\mathrm{ab}}, V_{\mathrm{C}_{\mathrm{f}}}^{\mathrm{bc}}, V_{\mathrm{C}_{\mathrm{f}}}^{\text {ac filter capacitance's voltage }} \\ \boldsymbol{x} & \text { state vector } \\ \boldsymbol{y} & \text { output matrix } \\ \omega & \text { angular velocity } \\ & \end{array}$

\section{Acknowledgement}

This research is supported by the National Research Development and Innovation Office (NKFIH) through grant No.115694. A. Magyar was supported by the János Bolyai Research Scholarship of the Hungarian Academy of Sciences.

\section{REFERENCES}

[1] Espelage, P.; Nowak, J.M.; Walker, L.H.: Symmetrical GTO current source inverter for wide speed range control of 2300 to $4160 \mathrm{~V}, 350$ to $7000 \mathrm{hp}$ induction motors, IEEE Ind. Appl. Soc. Conf. (IAS) 1988 1, 302-307 DOI 10.1109/IAS.1988.25079

[2] $\mathrm{Wu}, \mathrm{B}$.: High-power converters and AC drives (Wiley-IEEE Press, New Jersey, USA) 2006

[3] Ma, J.; Wu, B.; Rizzo, S.: A space vector modulated CSI-based AC drive for multi-motor applications, IEEE Trans. Power Electron., 1999 16(4), 535-544 DOI: 10.1109/APEC.1999.750460

[4] Wiseman, J.; Wu, B.; Castle, G.S.P.: A PWM current source rectifier with active damping for high power medium voltage applications, IEEE Power Electron. Spec. Conf. (PESC) 2002 1, 1930-1934 DOI: 10.1109/PSEC.2002.1023096

[5] Mariéthoz, S.; Morari, M.: Explicit ModelPredictive control of a PWM inverter with an LCL filter, IEEE Trans. Ind. Electron. 2008 56(2), 389399 DOI: 10.1109/TIE.2008.2008793

[6] Mariéthoz, S.; Almér, S.; Baja, M.; Beccuti, A.G.; Patino, D.; Wernrud, A.; Buisson, J.; Cormerais, H.; Geyer, T.; Fujioka, H.; Jonsson, U.T.; Kao, C.Y; Morari, M.; Papafotiou, G.; Rantzer, A.; Riedinger, P.: Comparison of hybrid control techniques for buck and boost DC-DC converters, IEEE Trans. Control Syst. Technol. 2010 18(5), 1126-1145 DOI: 10.1109/TCST.2009.2035306 